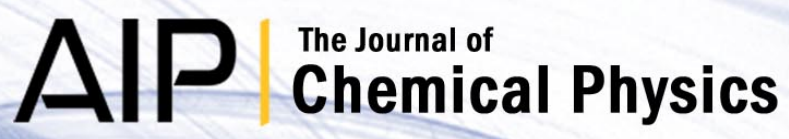

On the absolute thermodynamics of water from computer simulations: A comparison of first-principles molecular dynamics, reactive and empirical force fields

Tod A. Pascal, Daniel Schärf, Yousung Jung, and Thomas D. Kühne

Citation: J. Chem. Phys. 137, 244507 (2012); doi: 10.1063/1.4771974

View online: http://dx.doi.org/10.1063/1.4771974

View Table of Contents: http://jcp.aip.org/resource/1/JCPSA6/v137/i24

Published by the American Institute of Physics.

Additional information on J. Chem. Phys.

Journal Homepage: http://jcp.aip.org/

Journal Information: http://jcp.aip.org/about/about_the_journal

Top downloads: http://jcp.aip.org/features/most_downloaded

Information for Authors: http://jcp.aip.org/authors

Instruments for advanced science
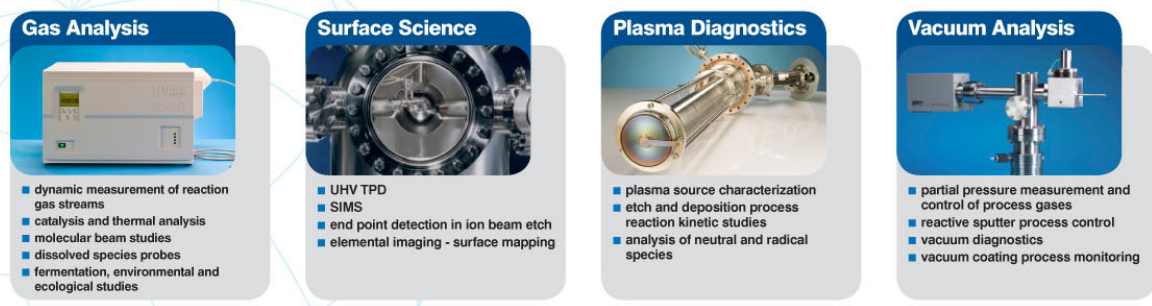

contact Hiden Analytical for further details

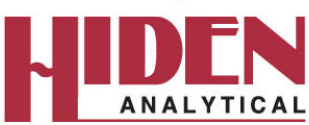

info@hideninc.com www.HidenAnalytical.com CLICK to view our product catalogue 


\title{
On the absolute thermodynamics of water from computer simulations: A comparison of first-principles molecular dynamics, reactive and empirical force fields
}

\author{
Tod A. Pascal, ${ }^{1,2, a)}$ Daniel Schärf, ${ }^{3}$ Yousung Jung, ${ }^{2}$ and Thomas D. Kühne ${ }^{3, b)}$ \\ ${ }^{1}$ Materials and Simulation Process Center, California Institute of Technology, Pasadena, \\ California 91125, USA \\ ${ }^{2}$ Graduate School of EEWS, Korea Advanced Institute of Science and Technology (KAIST), Daejeon, Korea \\ ${ }^{3}$ Institute of Physical Chemistry and Center of Computational Sciences, Johannes Gutenberg University \\ Mainz, Staudinger Weg 7, D-55128 Mainz, Germany
}

(Received 11 September 2012; accepted 29 November 2012; published online 28 December 2012)

\begin{abstract}
We present the absolute enthalpy, entropy, heat capacity, and free energy of liquid water at ambient conditions calculated by the two-phase thermodynamic method applied to ab initio, reactive and classical molecular dynamics simulations. We find that the absolute entropy and heat capacity of liquid water from $a b$ initio molecular dynamics (AIMD) is underestimated, but falls within the range of the flexible empirical as well as the reactive force fields. The origin of the low absolute entropy of liquid water from AIMD simulations is due to an underestimation of the translational entropy by $20 \%$ and the rotational entropy by $40 \%$ compared to the TIP3P classical water model, consistent with previous studies that reports low diffusivity and increased ordering of liquid water from AIMD simulations. Classical MD simulations with rigid water models tend to be in better agreement with experiment (in particular TIP3P yielding the best agreement), although the TIP4P-ice water model, the only empirical force field that reproduces the experimental melting temperature, has the lowest entropy, perhaps expectedly. This reiterates the limitations of existing empirical water models in simultaneously capturing the thermodynamics of solid and liquid phases. We find that the quantum corrections to heat capacity of water can be as large as $60 \%$. Although certain water models are computed to yield good absolute free energies of water compared to experiments, they are often due to the fortuitous enthalpy-entropy cancellation, but not necessarily due to the correct descriptions of enthalpy and entropy separately. @ 2012 American Institute of Physics. [http://dx.doi.org/10.1063/1.4771974]
\end{abstract}

\section{INTRODUCTION}

There has been great theoretical interest in calculating the absolute entropy, enthalpy, heat capacity, and free energy of the pure liquid, since it serves as a convenient reference point for understanding its various interfacial ${ }^{1}$ and temperature dependent properties. Numerous methods have been developed to calculate these thermodynamic properties, including very accurate methods based on perturbation theory, Kirkwood-Zwanzig thermodynamic integration, and Widom particle insertion. ${ }^{2}$ However, the large computational demands render these methods impractical for all but the most modest of systems. ${ }^{3}$

In particular, these methods are impractical for density functional theory (DFT) based Born-Oppenheimer (BOMD) or Car-Parrinello molecular dynamics (MD) simulations. Although these DFT-MD methods overcome many of the deficiencies of classical MD simulations based on empirical potentials by explicitly accounting for the electrons onthe-fly during the dynamics, they also come at a significant additional computational cost. Even the recently devised "Car-Parrinello-like approach to Born-Oppenheimer MD" method of Kühne et al. ${ }^{4}$ (aptly named AIMD),

\footnotetext{
a)Electronic mail: tpascal@wag.caltech.edu.

b) Electronic mail: kuehne@uni-mainz.de.
}

which allows for efficient and accurate first principles MD simulations at a much reduced computational complexity, would be outside the feasible range of the aforementioned methods.

One scheme to facilitate efficient calculations of thermodynamic observables from MD simulations is the two-phase thermodynamics method ${ }^{5,6}(2 \mathrm{PT})$. The $2 \mathrm{PT}$ method relies on approximating the partition function by evaluating the power spectrum (vibrational density of states function), as obtained by the temporal Fourier transform of the atomic velocity autocorrelation function. Due to frequent molecular collisions these correlation functions decay to zero very quickly, ${ }^{7}$ meaning that the thermodynamic observables can be accurately determined from a MD trajectory as short as $5 \mathrm{ps}$. The details of the 2PT method are presented in Refs. 5 and 6 and outlined in Sec. II. The 2PT method has been shown to be accurate and efficient in determining the entropy of pure liquids from classical MD simulations of bulk water in good agreement with more expensive method ${ }^{5,6}$ and to access the thermodynamics of water under hydrophobic confinement and surfaces. ${ }^{8}$ It has also recently been applied to understanding the thermodynamics of hydrocarbons at high temperatures and pressures and of metal alloys using BOMD simulations. ${ }^{9}$ A closely related technique to calculate the entropy based on the partial pair correlation function has been recently devised by Chakravarty and co-workers. ${ }^{10}$ 
Here, we exploit the efficiency of the 2PT method to provide a common basis for comparing the absolute thermodynamics across AIMD, reactive and classical molecular dynamics trajectories. While previous studies have calculated the excess thermodynamics ${ }^{11,12}$ and absolute entropy ${ }^{13}$ of liquid water from first principles simulations on rather short length and time scales (32-96 molecules for at most $30 \mathrm{ps),}$ our work represents the most extensive study of all the thermodynamic quantities for liquid water.

\section{METHODS}

\section{A. The 2PT method}

\section{Application to single-particle homogenous liquids}

If one considers the canonical (NVT) ensemble, the total system thermodynamics can be calculated from the partition function $Q$ by ${ }^{14}$

$$
\begin{gathered}
S=k_{b} T \frac{\partial \ln Q}{\partial T}+k_{b} \ln Q, \\
E=k_{b} T^{2} \frac{\partial \ln Q}{\partial T}, \\
C_{v}=\frac{\partial E}{\partial T}, \\
A=H-T S=-k_{b} T \ln Q,
\end{gathered}
$$

where $S$ is the entropy, $E$ the internal energy, $C_{v}$ the constant volume heat capacity, $A$ the Helmholtz free energy, and $k_{b}$ the Boltzmann constant. In the harmonic approximation, one approximates the normal modes of the system to be a set of $3 \mathrm{~N}$ harmonic oscillators, so that the partition function $Q$ can be expressed in term of the partition function $q_{i}$ for the individual modes. ${ }^{15}$ For a continuous distribution of normal modes, the partition function $Q$ can be shown to be related to $\operatorname{DoS}(v)$ by ${ }^{15,16}$

$$
\ln Q=\int_{0}^{\infty} \operatorname{DoS}(v) W(v) d v,
$$

where $W(v)$ is the relevant weighting function. Here, the $\operatorname{DoS}(v)$ is obtained from the Fourier transform of the atomic velocity autocorrelation functions

$$
\operatorname{DoS}(v)=\lim _{\tau \rightarrow \infty} \frac{1}{2 k T} \int_{-\tau}^{\tau} \sum_{j=1}^{N} \sum_{k=1}^{3} m_{j}\left\langle v_{j}^{k}\left(t^{\prime}+t\right) \cdot v_{j}^{k}(t)\right\rangle,
$$

where $\left\langle v_{j}^{k}(t \prime+t) \cdot v_{j}^{k}(t)\right\rangle$ is the $k$ th component of the velocity autocorrelation function of atom $j$ at time $t$. Physically, $\operatorname{DoS}(v)$ represents the density of normal modes of the system at frequency $v$.

Applying quantum statistics, ${ }^{14}$ i.e., assuming each mode to be a quantum harmonic oscillator vibrating with frequency $v$, the weighting functions are

$$
\begin{gathered}
W_{S}(v)=k_{b}\left(\frac{u}{\exp (u)-1}-\ln [1-\exp (-u)]\right), \\
W_{E}(v)=k_{b} T\left[\frac{u}{2}+\frac{u}{\exp (u)-1}\right],
\end{gathered}
$$

$$
\begin{gathered}
W_{C_{v}}(v)=k_{b}\left[\frac{u^{2} e^{u}}{\left(1-e^{u}\right)^{2}}\right], \\
W_{A}(v)=k_{b} T \ln \left[\frac{1-\exp (-u)}{\exp (-u / 2)}\right] .
\end{gathered}
$$

In a liquid, the $\operatorname{DoS}(v)$ has a finite value at $v=0$ due to diffusion: $\operatorname{DoS}(0)=12 \mathrm{MD} / k_{B} T$, where $D$ is the diffusion coefficient, and $M$ is the total mass of the corresponding system. Consequently, obtaining the thermodynamics by directly applying Eq. (2) in conjunction with the quantum weighting function of Eq. (4), would directly result in a singularity (positive infinity). Thus, borrowing on an idea as first proposed by Eyring and Ree, ${ }^{17}$ the $2 \mathrm{PT}$ method avoids this singularity by relating the $\operatorname{DoS}(v)$ of a liquid to two reference systems whose thermodynamics are easily calculated:

1. $\operatorname{DoS}_{\text {solid }}(v)$, which is the density of states of a solid at the same temperature and pressure. Here, $\lim _{v \rightarrow 0} D o S_{\text {solid }}(v)=0$. The thermodynamics of this reference system is obtained by substituting $D o S_{\text {solid }}(v)$ into Eq. (2) with weighting functions from Eq. (4).

2. $\operatorname{DoS}_{H S}(v)$, which is the density of states of a hardsphere gas at the same temperature and density. Here, $D o S_{H S}(0)=D o S(0)$ and $\lim _{v \rightarrow \infty} D o S_{H S}(v)=0 .{ }^{14}$ In practice, $D o S_{H S}(v)$ vanishes within a few hundred wavenumbers. The thermodynamics of this reference system is obtained by substituting $\operatorname{DoS}_{H S}(v)$ into Eq. (2) with weighing functions from the accurate Carnahan-Starling equation of state ${ }^{18}$

$$
\begin{gathered}
S=k_{b}\left(S^{i d}+\ln [z(f y)]+\frac{f y(3 f y-4)}{(1-f y)^{2}}\right), \\
E=0.5, \\
C_{v}=0.5, \\
A=E-T S,
\end{gathered}
$$

where $S^{\text {id }}$ is the entropy of an ideal gas at the same temperature and density, while $z(y)$ denotes the compressibility

$$
z(y)=\frac{1+y+y^{2}-y^{3}}{(1-y)^{3}} .
$$

Thus, in the 2PT method, the thermodynamics of a liquid is approximated as a linear combination of the thermodynamics of a hard-sphere gas and that of a solid, for example, the total entropy $S_{t o t}$ is

$$
S_{\text {tot }}=(1-f) S_{\text {solid }}+f S_{H S},
$$

where $f$ (the fluidicity factor) is the fraction of the $3 \mathrm{~N}$ degrees of freedom that are diffusive. Lin, Blanco, and Goddard ${ }^{6}$ showed that $f$ can be calculated selfconsistently from the MD trajectory by comparing the self-diffusion constant of the system to that of a hardsphere gas at the same temperature and density. They 
found a fundamental relation between $f$ and the dimensionless diffusivity $\Delta^{6}: 2 \Delta^{-9 / 2} f^{15 / 2}-6 \Delta^{-3} f^{7 / 2}$ $+6 \Delta^{-3 / 2} f^{2}+2(f-1)=0$.

\section{Application to molecular liquids}

The 2PT method relies solely on atomic velocities, meaning that it can be easily extended to molecular systems by logical grouping of the atomic velocities, provided that the various types of molecular motions are treated independently:

a. Translations The translational contribution $\left(v_{\text {trans }}\right)$ to the total velocity is obtained from the center of mass velocity

$$
V_{\text {trans }}(i)=\frac{1}{M_{i}} \sum_{k=1}^{3} \sum_{j} m_{j} V_{j}^{k},
$$

where $k$ is the $k t h$ component of the velocity vector of atom $j$ in molecule $i$. The translational thermodynamics is then obtained by considering only $v_{\text {trans }}$ in Eq. (3) to obtain $\operatorname{DoS}^{\text {trans }}(v)$ and applying the 2PT method as outlined before.

b. Rotations The rotational contribution $\left(V_{r o t}\right)$ is obtained by calculating the molecular angular velocity $\left(V_{a n g}\right)$

$$
\begin{aligned}
V_{r o t}(i) & =\omega(i) \times V_{t o t}(i)=I_{i}^{-1} \times L(i), \\
L(i) & =\sum_{j} m_{j}\left(R_{j} \times V_{j}\right),
\end{aligned}
$$

where $I_{i}^{-1}$ is the inverse of the moment of inertial tensor for molecule $I, L(i)$ is the angular momentum and $R_{j}$ is the position of atom $j$ in molecule $i$. The rotational thermodynamics is then obtained by considering only $V_{r o t}$ in Eq. (3) to obtain $\operatorname{DoS}^{r o t}(v)$ and applying the 2PT method as before, with the exception of the rotational entropy, where $W_{S}(v)$ in Eq. (4.1) is replaced with that of a quantum rigid rotor

$$
W_{S}^{r o t}(v)=\frac{1}{3} \frac{S^{R}}{k}=\frac{1}{3} \ln \left[\frac{\pi^{0.5} e^{1.5}}{\sigma}\left(\frac{T^{3}}{\Theta_{A} \Theta_{B} \Theta_{C}}\right)^{1 / 2}\right],
$$

where $S^{R}$ is the rotation entropy of a rigid body with rotational temperature $\Theta_{A}=\frac{h^{2}}{8 \pi^{2} I_{A} k}$ and $\sigma$ is the rotational symmetry ( $\sigma$ $=2$ for water).

c. Internal vibrations The component of the total molecular velocity arising from internal vibrational motions (bond stretching and angle bending in the case of AIMD-PBE, ReaxFF and flexible empirical waters) is taken as the remaining velocity after subtracting the first two contributions

$$
V_{v i b}(i)=V_{\text {tot }}(i)-\left[V_{\text {trans }}(i)+V_{\text {rot }}(i)\right] \text {. }
$$

The vibrational thermodynamics is obtained by substituting $V_{v i b}$ into Eq. (3). Note that there is no need to apply the 2PT decomposition here since $\operatorname{DoS}_{v i b}(v)$ has no diffusional component (i.e., the fluidicity is zero).

\section{Quantum corrections to classical trajectories}

Instead of assuming quantum statistics in Eq. (4), DoS(v) can be evaluated using the classical harmonic oscillator weighting function, i.e.,

$$
\begin{gathered}
W_{E}=k_{b} T, \\
W_{C_{v}}=k_{b}, \\
W_{S}=k_{b}[1-\ln u], \\
W_{A}=k_{b} T \ln u .
\end{gathered}
$$

As pointed out by Wilson et al., ${ }^{15}$ it is thus possible to approximate the quantum corrections to classical MD trajectories as the numerical difference in the $\operatorname{DoS}(v)$ evaluated with Eqs. (3) and (4). ${ }^{15}$ Thus, the corrected internal energy and heat capacity of the system becomes

$$
\begin{aligned}
E & =\left\langle E_{M D}\right\rangle+\Delta E_{Q \rightarrow C} \\
& =\left\langle E_{M D}\right\rangle+k_{b} T \int \operatorname{DoS}(v)\left[\frac{u}{2}+\frac{u}{\exp (u)-1}-1\right] d v, \\
C_{v} & =C_{v}^{\text {classical }}+\Delta C v_{Q \rightarrow C} \\
& =\frac{\left\langle E_{M D}^{2}\right\rangle-\left\langle E_{m d}\right\rangle^{2}}{N k_{b} T^{2}}+k_{b} \int \operatorname{DoS}(v)\left[\frac{u^{2} e^{u}}{\left(1-e^{u}\right)^{2}}-1\right] d v,
\end{aligned}
$$

where the brackets $\langle\ldots\rangle$ represent the static average and $N$ is the number of moles. Thus, the quantum corrected enthalpy inherently includes zero-point energy and heat capacity effects and is in principle more directly comparable to experiments than the potential energy from the MD simulations.

\section{B. AIMD-PBE simulations}

For the AIMD-PBE simulation, we considered a cubic box consisting of 128 light water molecules at ambient conditions for at least 150 ps. The simulation has been performed using the "Car-Parrinello-like approach to BornOppenheimer MD" of Kühne et al. ${ }^{4}$ as implemented in the mixed Gaussian and plane wave code $^{19}$ CP2K/Quickstep. ${ }^{20}$ The computational details are identical to Ref. 7, i.e., we have employed a triple-basis set with two additional sets of polarization functions (TZV2P) ${ }^{21}$ and a density cutoff. The unknown exchange and correlation (XC) functional is substituted by the PBE generalized gradient approximation (GGA), ${ }^{22,33}$ while the interactions between the valence electrons and the ionic cores are described by hard, normconserving pseudopotentials. ${ }^{23}$ Due to the fact that liquid water is a wide bandgap insulator, the Brillouin zone is sampled at the $\Gamma$-point only. It should be pointed out that without any correction for London dispersion forces common GGA $\mathrm{XC}$ functionals underestimate the liquid water density by as much as $\sim 10 \%-20 \% .^{24}$ However, since the neglect of vander-Waals interactions primarily affects the density and only to a lesser extent the structure of liquid water, ${ }^{25}$ we have fixed the volume to recover the experimental density and performed our AIMD simulation in the canonical ensemble. 


\section{Classical and ReaxFF simulations}

Additionally, we performed classical simulations using the ReaxFF force field for liquid water ${ }^{26}$ and the SPC-Fw, F3C, IPBV, TIP4P/F, SPC, SPC/E, TIP3P, TIP4P-Ew, TIP4P2005, and TIP4P-ice water models ${ }^{27,28}$ using the LAMMPS ${ }^{29}$ code. We initiated these simulations from a pre-equilibrated cubic box of 512 water molecules. Newton's equations of motion are integrated in the constant pressure (1 bar)-constant temperature $(300 \mathrm{~K})$ or $N P T$ ensemble for at least $10 \mathrm{~ns}$. The temperature coupling constant was 0.1 ps while the pressure piston constant was $2.0 \mathrm{ps}$. The equations of motion used are those of Shinoda et al., ${ }^{30}$ which combine the hydrostatic equations of Martyna et al. ${ }^{31}$ with the strain energy proposed by Parrinello and Rahman. ${ }^{32}$ The time integration schemes closely follow the time-reversible measure-preserving Verlet integrators derived by Tuckerman et al. ${ }^{33}$ Range limited van-der-Waals interactions were truncated at $10 \AA$ by a cubic spline function, while the long range electrostatics were treated using the PPPM method ${ }^{34}$ for the simulations with empirical potentials and with a 7 th order taper function for the ReaxFF simulations. For the 2PT analysis, snapshots of the system was saved every 100 ps and subject to a further 10 ps of constant temperature, constant volume (NVT) dynamics, with coordinates and velocities saved every 4 ps.

\section{RESULTS AND DISCUSSIONS}

\section{A. Standard molar entropy of AIMD-PBE simulation}

As shown in Figure 1(a), the velocity autocorrelation functions of the hydrogen and oxygen atoms from our AIMDPBE simulations are rapidly decaying to zero within 800 fs. The resulting power spectrum of Figure 1(b) shows good agreement with the experimental $\mathrm{HOH}$ bending mode $\left(1643.5 \mathrm{~cm}^{-1}\right)$ and the $\mathrm{OH}$ stretching modes $\left(3404.0 \mathrm{~cm}^{-1}\right),{ }^{35}$ even displaying features consistent with the splitting of the symmetric and asymmetric stretching, which is due to distortions of the tetrahedral coordination of liquid water caused by finite temperature. ${ }^{10}$ As a measure of the precision of the 2PT method when applied to AIMD simulations, we note that the calculated standard molar entropy converges after 20-40 ps of dynamics (Figure 2(a)). We examined the dependence of the entropy on the length of trajectory used for the analysis, and found that converged results were obtained from $10 \mathrm{ps}$

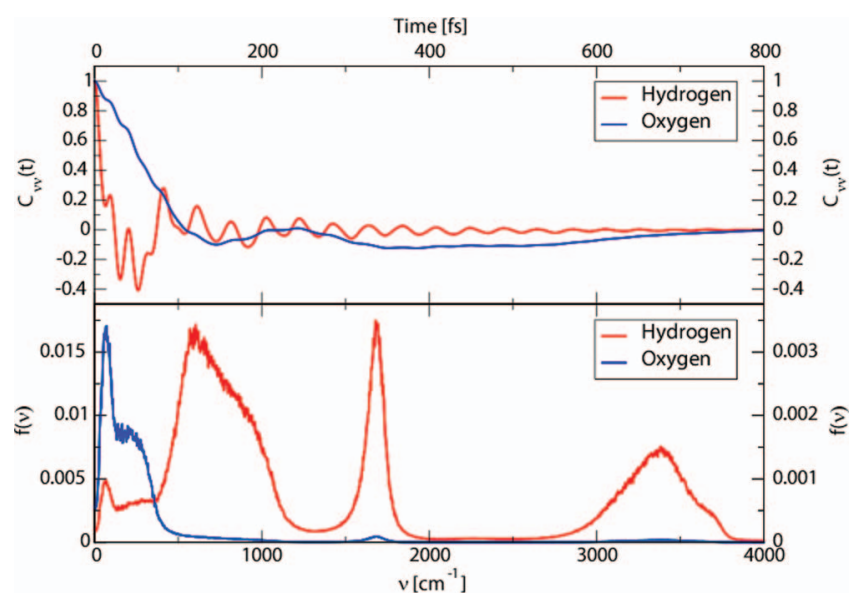

FIG. 1. (Top panel) Velocity autocorrelation function from AIMD. (Bottom panel) Associated power spectrum obtained by a temporal Fourier transform of the VACF.

trajectories (Figure 2(b)). The calculated entropy is 51.32 $\pm 1.79 \mathrm{~J} / \mathrm{mol} / \mathrm{K}$, as determined from 12 measurements over the final 120 ps trajectory in 10 ps windows. This verifies that the 2PT method is capable of predicting the thermodynamics on time and length scales accessible to AIMD simulations.

\section{B. Comparison to entropy of classical water model and ReaxFF}

In Table I, we present the absolute thermodynamics of our AIMD-PBE and ReaxFF simulations, as well as the results from various common empirical water models. We find that the standard molar entropy from AIMD-PBE (-27\%) and ReaxFF $(-13 \%)$ simulations, as well as the flexible $\mathrm{SPC} / \mathrm{FW}(-13 \%), \mathrm{F} 3 \mathrm{C}(-12 \%)$, IPBV $(-6 \%)$, and TIP4/F $(-16 \%)$ classical potentials are all underestimated when compared to experiment. The empirical rigid water models tend to have better agreement with experiment, ${ }^{36}$ with TIP3P $(+3 \%)$ being the best performer. We note that the recently developed TIP4P-2005 $(-18 \%)$ and TIP4P-ice $(-25 \%)$ force fields also underestimate the entropy compared to experiment and are in fact closer to our AIMD-PBE simulations. The relatively poor performance of the TIP4-2005 water model is somewhat surprising, especially since it is considered an improvement upon the parent TIP4P-Ew water model which only underestimates

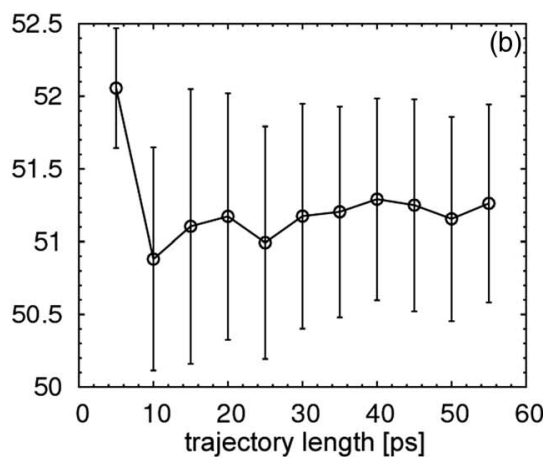

FIG. 2. (a) The total entropy of the AIMD simulation as a function of simulation time. We find convergence after 20 ps of MD. (b) Convergence in the entropy of the AIMD simulation with respect to the length of the trajectory. We find that 10 ps of dynamics are required to obtain converged results. 
TABLE I. Standard molar entropy $\left(S^{0}\right)$, enthalpy $\left(H^{0}\right)$, constant volume heat capacity with $\left(C_{v}{ }^{Q}\right)$ and without $\left(C_{v}{ }^{C}\right)$ quantum corrections, and Gibbs free energy $\left(G^{0}\right)$ of liquid water at $298 \mathrm{~K}$ from AIMD, ReaxFF MD and various classical MD simulations. The quoted values are statistical averages in $100 \mathrm{ps}$ intervals for the ReaxFF and classical MD trajectories and $10 \mathrm{ps}$ windows for the AIMD simulations. The potential energy from MD ( $E^{\text {Pot }}$ ) is also tabulated. The statistical uncertainty is quoted in brackets. The excess free energy $\left(\Delta G^{e x}\right)$ is also presented by subtracting the idea gas contributions.

\begin{tabular}{|c|c|c|c|c|c|c|c|}
\hline & $S^{0}(\mathrm{~J} / \mathrm{mol} / \mathrm{K})$ & $H^{0}(\mathrm{~kJ} / \mathrm{mol})$ & $E^{P o t}(\mathrm{~kJ} / \mathrm{mol})$ & $C_{v}{ }^{Q}(\mathrm{~J} / \mathrm{mol} / \mathrm{K})$ & $C_{v}{ }^{C}(\mathrm{~J} / \mathrm{mol} / \mathrm{K})$ & $G^{0}(\mathrm{~kJ} / \mathrm{mol})$ & $\Delta G^{e x}(\mathrm{~kJ} / \mathrm{mol})$ \\
\hline Experiment & $69.9^{\mathrm{a}}$ & $-34.1^{\mathrm{b}}$ & & 74.5 & & -55.9 & -24 \\
\hline AIMD-PBE & $\begin{array}{l}51.32 \\
(1.79)\end{array}$ & $\begin{array}{r}-43.62 \\
(2.69)\end{array}$ & $\begin{array}{r}-46.32 \\
(2.52)\end{array}$ & $\begin{array}{l}51.36 \\
(3.17)\end{array}$ & $\begin{array}{l}89.34 \\
(3.20)\end{array}$ & $\begin{array}{r}-58.91 \\
(2.40)\end{array}$ & -27.01 \\
\hline ReaxFF & $\begin{array}{c}61.47 \\
(0.84)\end{array}$ & $\begin{array}{r}-31.68 \\
(0.21)\end{array}$ & $\begin{array}{r}-33.98 \\
(0.31)\end{array}$ & $\begin{array}{l}72.17 \\
(2.56)\end{array}$ & $\begin{array}{c}107.61 \\
(2.53)\end{array}$ & $\begin{array}{r}-49.98 \\
(0.27)\end{array}$ & -19.08 \\
\hline \multicolumn{8}{|c|}{ Flexible water models } \\
\hline SPC-Fw & $\begin{array}{l}61.36 \\
(0.38)\end{array}$ & $\begin{array}{r}-30.60 \\
(0.15)\end{array}$ & $\begin{array}{r}-33.01 \\
(0.20)\end{array}$ & $\begin{array}{l}66.61 \\
(1.38)\end{array}$ & $\begin{array}{r}108.68 \\
(1.42)\end{array}$ & $\begin{array}{r}-48.89 \\
(0.66)\end{array}$ & -17.99 \\
\hline $\mathrm{F} 3 \mathrm{C}$ & $\begin{array}{l}61.70 \\
(0.29)\end{array}$ & $\begin{array}{r}-30.48 \\
(0.18)\end{array}$ & $\begin{array}{r}-32.90 \\
(0.16)\end{array}$ & $\begin{array}{l}68.53 \\
(1.26)\end{array}$ & $\begin{array}{c}103.84 \\
(1.31)\end{array}$ & $\begin{array}{r}-48.81 \\
(0.25)\end{array}$ & -17.91 \\
\hline IPBV & $\begin{array}{l}65.97 \\
(0.91)\end{array}$ & $\begin{array}{r}-32.71 \\
(0.11)\end{array}$ & $\begin{array}{r}-34.89 \\
(0.15)\end{array}$ & $\begin{array}{l}62.56 \\
(0.37)\end{array}$ & $\begin{array}{c}96.34 \\
(1.93)\end{array}$ & $\begin{array}{r}-52.37 \\
(0.37)\end{array}$ & -20.47 \\
\hline TIP4P/F & $\begin{array}{l}58.75 \\
(0.77)\end{array}$ & $\begin{array}{r}-35.41 \\
(0.12)\end{array}$ & $\begin{array}{r}-37.72 \\
(0.24)\end{array}$ & $\begin{array}{l}60.41 \\
(1.80)\end{array}$ & $\begin{array}{l}90.67 \\
(2.53)\end{array}$ & $\begin{array}{r}-52.92 \\
(0.56)\end{array}$ & -21.02 \\
\hline \multicolumn{8}{|c|}{ Rigid water models } \\
\hline $\mathrm{SPC}$ & $\begin{array}{l}65.74 \\
(0.19)\end{array}$ & $\begin{array}{r}-32.26 \\
(0.12)\end{array}$ & $\begin{array}{r}-33.70 \\
(0.16)\end{array}$ & $\begin{array}{c}65.03 \\
(0.83)\end{array}$ & $\begin{array}{l}75.27 \\
(0.87)\end{array}$ & $\begin{array}{r}-51.61 \\
(0.13)\end{array}$ & -20.71 \\
\hline $\mathrm{SPC} / \mathrm{E}^{\mathrm{c}}$ & $\begin{array}{l}60.30 \\
(0.25)\end{array}$ & $\begin{array}{r}-36.79 \\
(0.11)\end{array}$ & $\begin{array}{r}-37.80 \\
(0.15)\end{array}$ & $\begin{array}{l}75.86 \\
(0.89)\end{array}$ & 86.48 & $\begin{array}{r}-49.57 \\
(0.13)\end{array}$ & -23.67 \\
\hline TIP3P & $\begin{array}{l}72.51 \\
(0.27)\end{array}$ & $\begin{array}{r}-33.44 \\
(0.17)\end{array}$ & $\begin{array}{r}-34.29 \\
(0.22)\end{array}$ & $\begin{array}{l}57.47 \\
(0.67)\end{array}$ & $\begin{array}{l}66.70 \\
(0.72)\end{array}$ & $\begin{array}{r}-54.74 \\
(0.10)\end{array}$ & -23.84 \\
\hline TIP4P-Ew & $\begin{array}{l}64.33 \\
(0.25)\end{array}$ & $\begin{array}{r}-30.81 \\
(0.11)\end{array}$ & $\begin{array}{r}-32.50 \\
(0.12)\end{array}$ & $\begin{array}{l}68.21 \\
(0.97)\end{array}$ & $\begin{array}{l}80.00 \\
(1.00)\end{array}$ & $\begin{array}{r}-49.98 \\
(0.15)\end{array}$ & -19.08 \\
\hline TIP4P-2005 & $\begin{array}{l}57.47 \\
(0.31)\end{array}$ & $\begin{array}{r}-37.29 \\
(0.14)\end{array}$ & $\begin{array}{r}-38.77 \\
(0.15)\end{array}$ & $\begin{array}{l}76.65 \\
(1.37)\end{array}$ & $\begin{array}{l}84.71 \\
(1.15)\end{array}$ & $\begin{array}{r}-54.24 \\
(0.12)\end{array}$ & -23.44 \\
\hline TIP4P-ice ${ }^{\mathrm{e}}$ & $\begin{array}{l}52.41 \\
(0.27)\end{array}$ & $\begin{array}{r}-40.60 \\
(0.15)\end{array}$ & $\begin{array}{r}-41.74 \\
(0.16)\end{array}$ & $\begin{array}{l}80.71 \\
(1.19)\end{array}$ & $\begin{array}{l}85.67 \\
(1.56)\end{array}$ & $\begin{array}{r}-56.27 \\
(0.19)\end{array}$ & -25.37 \\
\hline
\end{tabular}

a Reference 39 .

${ }^{\mathrm{b}}$ Reference 40 gives $\Delta H=H^{0}-4 \mathrm{RT}$.

${ }^{\mathrm{c}}$ Includes dipolar correction of $5.22 / \mathrm{kJ} \mathrm{mol}^{-1}$ and $4.37 / \mathrm{kJ} \mathrm{mol}^{-1}$.

${ }^{\mathrm{d}}$ Includes dipolar correction of $4.60 / \mathrm{kJ} \mathrm{mol}^{-1}$.

${ }^{\mathrm{e}}$ Includes dipolar correction of $6.90 / \mathrm{kJ} \mathrm{mol}^{-1}$.

the entropy by $-8 \%$ compared to experiment. On the other hand, the TIP4P-ice water model, the only empirical potential in our dataset that reproduces the melting point of ice by strengthening of the hydrogen bond interactions, can be expected to induce unrealistic ordering in liquid water, similar to GGA XC functionals within DFT. ${ }^{13}$

\section{Quantum corrections and the liquid enthalpy and heat capacity}

The quantum corrections to the enthalpy (Table I) range from +0.85 (TIP3P) to +2.42 (IPBV) kJ/mol, with $\sim 1.25 \mathrm{~kJ} /$ mol corrections for the rigid empirical water models. The AIMD-PBE, ReaxFF, and flexible water models have greater $>2 \mathrm{~kJ} / \mathrm{mol}$ corrections. In general, these corrections improve the agreement between the calculated enthalpies and experiment. Nevertheless, our corrected AIMD-PBE liquid enthalpy is $-43.62 \mathrm{~kJ} / \mathrm{mol}$, or $28 \%$ larger than experiment. In comparison, the ReaxFF and various empirical water models show better agreement with experiment, with errors between $-9 \%$ (SPC-Fw) and +9\% (TIP4P-2005). Of course, the ReaxFF and classical force fields have been parameterized to reproduce the heat of vaporization of the liquid so that their relatively good performance in reproducing the liquid enthalpy is unsurprising. The single major outlier among the empirical potentials is the TIP4-ice water model, which overestimates the enthalpy by $+19 \%$. This lends further evidence that there is no combination of parameters, which allows classical, non-polarizable two-body water models to simultaneously describe the properties of the bulk, liquid, and ice. $^{28}$

We find that quantum corrections lower the constant volume heat capacity and are critical for accurately comparing the flexible water models to experiment. For example, the uncorrected heat capacity of the SPC-Fw water model is $108.68 \mathrm{~J} / \mathrm{mol} / \mathrm{K}$ while the quantum corrected value is $68.53 \mathrm{~J} / \mathrm{mol} / \mathrm{K}$, in much better agreement to the experimental value of $74.5 \mathrm{~J} / \mathrm{mol} / \mathrm{K}$. Among the empirical water models, we find the SPC-Fw and rigid SPC/E and TIP4P-2005 water models to be the best performers, with errors of $\sim+2 \%$. The heat capacity of our AIMD-PBE simulation is $51.36 \mathrm{~J} / \mathrm{mol} / \mathrm{K}$, or $30 \%$ underestimated compared to experiments. This suggests that AIMD-PBE will exhibit poor temperature dependent heats of vaporization, thus care should be taken when attempting to approximate the $300 \mathrm{~K}$ thermodynamic properties from AIMD simulations run at higher temperatures. 
TABLE II. Components of the standard molar entropy $(\mathrm{J} / \mathrm{mol} / \mathrm{K})$ of liquid water at $298 \mathrm{~K}$ from various levels of theory and classical force fields. Here, we define the total translational entropy $S_{\text {trans }}=S_{\text {diffuse }}+S_{\text {lib-trans }}$.

\begin{tabular}{|c|c|c|c|c|c|c|}
\hline & $S_{\text {total }}^{0}$ & $S_{\text {diffuse }}^{0}$ & $S_{\text {lib-trans }}^{0}$ & $S_{\text {trans }}^{0}$ & $S_{r o t}^{0}$ & $S_{v i b}^{0}{ }^{\mathrm{a}}$ \\
\hline AIMD-PBE (This work) & $51.32(1.79)$ & $1.59(0.27)$ & $42.55(1.33)$ & $44.05(1.55)$ & $7.21(0.49)$ & 0.05 \\
\hline BOMD-PBE ${ }^{b}$ & $42.74(0.33)$ & & & $34.84(0.25)$ & $7.60(0.08)$ & 0.30 \\
\hline BOMD-DRSLLPBE $^{b}$ & $52.47(0.50)$ & & & $42.18(0.45)$ & $8.79(0.05)$ & 0.24 \\
\hline ReaxFF & $61.47(0.84)$ & $1.79(0.09)$ & $47.49(0.29)$ & $49.28(0.27)$ & $12.28(0.16)$ & 0.04 \\
\hline SPC-Fw & $61.36(0.38)$ & $2.30(0.11)$ & $47.58(0.35)$ & $49.87(0.40)$ & $11.01(0.15)$ & 0.09 \\
\hline $\mathrm{F} 3 \mathrm{C}$ & $61.70(0.29)$ & $2.48(0.06)$ & $47.72(0.28)$ & $50.12(0.33)$ & $11.55(0.11)$ & 0.04 \\
\hline IPBV & $65.97(0.91)$ & $3.03(0.30)$ & $50.37(0.58)$ & $53.41(0.87)$ & $11.93(0.14)$ & 0.63 \\
\hline TIP4/F & $58.75(0.77)$ & $2.44(0.22)$ & $46.90(0.48)$ & $49.34(0.66)$ & $9.35(0.17)$ & 0.06 \\
\hline SPC & $65.74(0.19)$ & $2.94(0.07)$ & $50.54(0.22)$ & $53.48(0.25)$ & $12.19(0.04)$ & $\ldots$ \\
\hline SPC/E & $60.30(0.25)$ & $2.31(0.06)$ & $47.58(0.23)$ & $49.89(0.26)$ & $10.50(0.06)$ & $\ldots$ \\
\hline TIP3P & $72.51(0.27)$ & $3.83(0.07)$ & $54.22(0.27)$ & $58.05(0.30)$ & $14.33(0.07)$ & $\ldots$ \\
\hline TIP4P & $64.33(0.25)$ & $2.93(0.06)$ & $50.31(0.21)$ & $53.24(0.27)$ & $11.13(0.05)$ & $\ldots$ \\
\hline TIP4P-2005 & $57.47(0.31)$ & $2.08(0.05)$ & $46.20(0.24)$ & $48.28(0.29)$ & $9.14(0.06)$ & $\ldots$ \\
\hline TIP4P-ice & $52.41(0.27)$ & $1.49(0.07)$ & $43.15(0.27)$ & $44.64(0.34)$ & $7.76(0.05)$ & $\ldots$ \\
\hline
\end{tabular}

${ }^{\mathrm{a}}$ Uncertainties in $S^{0}{ }_{v i b}$ are $<0.001$.

${ }^{\mathrm{b}}$ Reference 13.

\section{Liquid free energy}

The liquid free energy of our AIMD-PBE simulation is $-58.91 \pm 2.4 \mathrm{~kJ} / \mathrm{mol}$, slightly larger than the experimental value of $-55.9 \mathrm{~kJ} / \mathrm{mol}$, though this results directly from a fortuitous cancellation of errors between the overestimated liquid enthalpy and the underestimated entropy. This is consistent with a previous study examining the components of the excess free energy of an AIMD simulation using the BLYP XC functional. ${ }^{12}$ However, our AIMD-PBE liquid free energy is higher than a previously reported value ${ }^{11}$ at $314 \mathrm{~K}$ of $-52.24 \mathrm{~kJ} / \mathrm{mol}$ using quasi-chemical theory, albeit for a more modest system (32 molecules) and simulation time ( $20 \mathrm{ps})$.

The classical water models exhibit better agreement to the experimental liquid free energy, although there is a general underestimation by $-11 \%$. Interestingly, the TIP4P-ice water model shows the best agreement with experiment liquid free energy $(+0.6 \%)$ though, as in the case of the AIMD simulation, this results from an underestimation of the entropy and an overestimation of the enthalpy. Indeed, our results highlight that there is no description of liquid water, whether classical or $a b$ initio, that simultaneously gives good agreement with the experimental entropy and enthalpy.

\section{E. Components of liquid entropy}

We gain further insight into the nature of the entropic underestimation of the AIMD-PBE XC functional by partitioning the total absolute entropy into contributions arising from diffusion $S_{\text {diffuse }}$, librational motions, i.e., translations $S_{\text {lib-trans }}$ and rotations $S_{r o t}$, and internal vibrations $S_{v i b}$. Note that the more familiar translational entropy (Sec. II A 2) $S_{\text {trans }}$ $=S_{\text {diffuse }}+S_{\text {lib-trans }}$.

While there is no direct comparison to experiments, we note that the translational modes of water are 2-3 times softer than the rotational modes, so that one can reasonably expect two-thirds to three-quarters of the water entropy to arise from
$S_{\text {trans }}$. This is indeed the case for several of the empirical water potentials, in particular the SPC/E $\left(S_{\text {trans }} / S_{\text {total }}=83 \%\right)$ and the IPBV (79\%) water models (Table II). On the other hand, the AIMD-PBE (86\%) and TIP4P-ice (85\%) MD simulations have the highest translational/rotational entropy ratios, further evidence for its enhanced hydrogen bonding strength. The low diffusion constant of our AIMD-PBE simulations $(67 \%$ lower than experiment ${ }^{7}$ ) leads to only $16 \%$ of the translational modes being considered as diffusional (Table III) in the 2PT method. This is similar to the TIP4P-ice value of $20 \%$ and about half the value of the classical potentials, which range from $24 \%$ in the case of the TIP $4 / \mathrm{F}$ to $39 \%$ for the TIP3P water model. Thus, while low diffusivity contributes to the low translational entropy of the AIMD-PBE simulation, the major factors are restricted low frequency rattling motions induced by hydrogen bonding interactions that are too stiff.

TABLE III. Density $\rho$, self-diffusion constant $D$, and $\%$ of modes that are diffusional for liquid water at $298 \mathrm{~K}$ from AIMD, reactive and classical MD simulations.

\begin{tabular}{lccc}
\hline \hline & $\rho(\mathrm{g} / \mathrm{cc})$ & $D\left(\times 10^{5} \mathrm{~cm}^{2} / \mathrm{s}\right)$ & $\%$ diffusional modes \\
\hline Experiment $^{\mathrm{a}}$ & 0.997 & 2.27 & \\
AIMD-PBE & $0.995(0.013)$ & $1.15(0.30)$ & $15.79(2.34)$ \\
ReaxFF & $0.988(0.012)$ & $1.91(0.02)$ & $19.46(1.28)$ \\
SPC-Fw & $1.000(0.014)$ & $2.54(0.27)$ & $28.57(1.48)$ \\
F3C & $0.994(0.016)$ & $2.93(0.24)$ & $30.67(1.20)$ \\
IPBV & $0.985(0.024)$ & $4.68(1.23)$ & $30.89(3.86)$ \\
TIP4/F & $0.996(0.030)$ & $2.82(0.47)$ & $24.35(2.08)$ \\
SPC & $0.968(0.019)$ & $4.11(0.37)$ & $35.80(1.40)$ \\
SPC/E & $0.984(0.019)$ & $2.60(0.24)$ & $28.61(1.22)$ \\
TIP3P & $1.022(0.020)$ & $5.69(0.21)$ & $38.90(1.41)$ \\
TIP4P & $0.970(0.018)$ & $4.01(0.35)$ & $34.95(1.40)$ \\
TIP4P-2005 & $0.987(0.019)$ & $2.16(0.23)$ & $25.80(1.31)$ \\
TIP4P-ice & $0.982(0.015)$ & $1.25(0.17)$ & $19.54(1.36)$ \\
\hline \hline
\end{tabular}

${ }^{a}$ Reference 41. 


\section{CONCLUSIONS AND OUTLOOK}

We have presented a thorough analysis of a long DFTMD trajectory performed with an efficient method of extracting absolute entropies and quantum corrections to the enthalpy and heat capacity. Our simulation methodology allows for convergence in the thermodynamic properties to be obtained after only $40 \mathrm{ps}$ of dynamics using as short as $10 \mathrm{ps}$ of sampling. While the calculated liquid free energy is in reasonable agreement with experiment, this arises from fortuitous cancelling of error between the enhanced enthalpy and reduced entropy of the DFT-MD simulation. Further, by comparing the thermodynamics to standard reactive and classical water models, we have discovered that the low entropy of DFT-MD simulation arises from suppressed low frequency librational motions with overemphasized H-bonding. Thus, one possible route to obtain DFT-MD liquid water simulations with improved thermodynamic properties would be either to use hybrid DFT XC functional that consists of a fraction of exact exchange to partially correct for overdelocalized states in GGA and/or the inclusion of nuclear quantum effects. ${ }^{37}$ Although the enormous additional computational cost of the latter methods would limit investigations to relatively small systems and/or simulation times, the efficiency of the 2PT method as shown here demonstrates that such investigations can be feasible. The liquid thermodynamics may also be improved by inclusion of empirical dispersion functions, as a previous study has shown that this is required in order to obtain the correct density of liquid water at $300 \mathrm{~K} .^{38}$

\section{ACKNOWLEDGMENTS}

This work was partially supported by the World Class University program (R31-2008-000-10055-0) of Korea; Energy, Environment, Water, and Sustainability Initiative funding from KAIST. D.S. would like to thank the Max Planck Graduate Center. T.D.K. acknowledges financial support from the MAINZ Graduate School of Excellence and the IDEE project of the Carl Zeiss Foundation.

${ }^{1}$ A. Kalra, S. Garde and G. Hummer, Proc. Natl. Acad. Sci. U.S.A. 100(18), 10175 (2003); F. N. Kemmer, Water: The Universal Solvent (Nalco Chemical, Oak Brook, IL, 1977); P. Ball, Chem. Rev. 108(1), 74 (2008); S. Narayan, J. Muldoon, M. G. Finn, V. V. Fokin, H. C. Kolb, and K. B. Sharpless, Angew. Chem., Int. Ed. 44(21), 3275 (2005).

${ }^{2}$ X. Kong and C. L. Brooks, III, J. Chem. Phys. 105(6), 2414 (1996); J. G. Kirkwood, ibid. 3(5), 300 (1935); R. Zwanzig, ibid. 22(8), 1420 (1954); F. Schmid and T. Schilling, Phys. Procedia 4(0), 131 (2010); B. Widom, J. Chem. Phys. 39(11), 2808 (1963).

${ }^{3}$ D. Frenkel and B. Smit, Understanding Molecular Simulation: From Algorithms to Applications (Academic, San Diego, CA, 2002).

${ }^{4}$ T. D. Kühne, M. Krack, F. R. Mohamed, and M. Parrinello, Phys. Rev. Lett. 98(6), 066401 (2007).

${ }^{5}$ S. T. Lin, P. K. Maiti, and W. A. Goddard, J. Phys. Chem. B 114(24), 8191 (2010); T. A. Pascal, S. T. Lin, and W. A. Goddard, III, Phys. Chem. Chem. Phys. 13(1), 169 (2011).

${ }^{6}$ S. T. Lin, M. Blanco, and W. A. Goddard, J. Chem. Phys. 119(22), 11792 (2003).

${ }^{7}$ T. D. Kühne, M. Krack, and M. Parrinello, J. Chem. Theory Comput. 5(2), 235 (2009).

${ }^{8}$ H. Kumar, B. Mukherjee, S. T. Lin, C. Dasgupta, A. K. Sood, and P. K. Maiti, J. Chem. Phys. 134(12), 124105 (2011); T. A. Pascal, W. A. God- dard, and Y. Jung, Proc. Natl. Acad. Sci. U.S.A. 108(29), 11794 (2011); T. A. Pascal, S.-T. Lin, W. Goddard, and Y. Jung, J. Phys. Chem. Lett. 3(3), 294 (2012).

${ }^{9}$ L. Spanu, D. Donadio, D. Hohl, E. Schwegler, and G. Galli, Proc. Natl. Acad. Sci. U.S.A. 108(17), 6843 (2011); A. M. Teweldeberhan and S. A. Bonev, Phys. Rev. B 83(13), 134120 (2011).

${ }^{10}$ R. Sharma, M. Agarwal, and C. Chakravarty, Mol. Phys. 106(15), 1925 (2008).

${ }^{11}$ D. Asthagiri, L. R. Pratt, and J. D. Kress, Phys. Rev. E 68(4), 041505 (2003).

${ }^{12}$ V. Weber and D. Asthagiri, J. Chem. Phys. 133(14), 141101 (2010).

${ }^{13}$ C. Zhang, L. Spanu, and G. Galli, J. Phys. Chem. B 115(48), 14190 (2011).

${ }^{14}$ D. A. McQuarrie, Statistical Mechanics (University Science Books, Sausalito, CA, 2000)

${ }^{15}$ P. H. Berens, D. H. J. Mackay, G. M. White, and K. R. Wilson, J. Chem. Phys. 79(5), 2375 (1983).

${ }^{16}$ A. Pohorille, L. R. Pratt, R. A. LaViolette, M. A. Wilson, and R. D. MacElroy, J. Chem. Phys. 87(10), 6070 (1987).

${ }^{17}$ H. Eyring and T. Ree, Proc. Natl. Acad. Sci. U.S.A. 47(4), 526 (1961).

${ }^{18}$ N. F. Carnahan and K. E. Starling, J. Chem. Phys. 53(2), 600 (1970).

${ }^{19}$ G. Lippert, J. Hutter, and M. Parrinello, Mol. Phys. 92(3), 477 (1997).

${ }^{20}$ J. VandeVondele, M. Krack, F. Mohamed, M. Parrinello, T. Chassaing, and J. Hutter, Comput. Phys. Commun. 167(2), 103 (2005).

${ }^{21}$ J. VandeVondele and J. Hutter, J. Chem. Phys. 127(11), 114105 (2007).

${ }^{22}$ J. P. Perdew, K. Burke, and M. Ernzerhof, Phys. Rev. Lett. 77(18), 3865 (1996).

${ }^{23}$ S. Goedecker, M. Teter, and J. Hutter, Phys. Rev. B 54(3), 1703 (1996); M. Krack, Theor. Chem. Acc. 114(1-3), 145 (2005).

${ }^{24}$ J. Schmidt, J. VandeVondele, I. F. W. Kuo, D. Sebastiani, J. I. Siepmann, J. Hutter, and C. J. Mundy, J. Phys. Chem. B 113(35), 11959 (2009); T. D. Kühne, T. A. Pascal, E. Kaxiras, and Y. Jung, J. Phys. Chem. Lett. 2(2), 105 (2011); Z. H. Ma, Y. L. Zhang, and M. E. Tuckerman, J. Chem. Phys. 137(4), 044506 (2012).

${ }^{25}$ I. C. Lin, A. P. Seitsonen, I. Tavernelli, and U. Rothlisberger, J. Chem. Theory Comput. 8(10), 3902 (2012).

${ }^{26}$ D. Raymand, A. C. T. van Duin, D. Spångberg, W. A. Goddard, III, and K. Hermansson, Surf. Sci. 604(9-10), 741 (2010).

${ }^{27}$ Y. Wu, H. L. Tepper, and G. A. Voth, J. Chem. Phys. 124(2), 024503 (2006); M. Levitt, M. Hirshberg, R. Sharon, K. E. Laidig, and V. Daggett, J. Phys. Chem. B 101(25), 5051 (1997); S. Izvekov, M. Parrinello, C. J. Burnham, and G. A. Voth, J. Chem. Phys. 120(23), 10896 (2004); M. W. Mahoney and W. L. Jorgensen, ibid. 115(23), 10758 (2001); J. Abascal and C. Vega, ibid. 123(23), 234505 (2005); H. J. C. Berendsen, J. R. Grigera, and T. P. Straatsma, J. Phys. Chem. 91(24), 6269 (1987); H. W. Horn, W. C. Swope, J. W. Pitera, J. D. Madura, T. J. Dick, G. L. Hura, and T. Head-Gordon, J. Chem. Phys. 120(20), 9665 (2004).

${ }^{28}$ J. Abascal, E. Sanz, R. Fernandez, and C. Vega, J. Chem. Phys. 122(23), 234511 (2005).

${ }^{29}$ S. Plimpton, J. Comput. Phys. 117(1), 1 (1995).

${ }^{30}$ W. Shinoda, M. Shiga, and M. Mikami, Phys. Rev. B 69(13), 134104 (2004).

${ }^{31}$ G. J. Martyna, D. J. Tobias, and M. L. Klein, J. Chem. Phys. 101(5), 4177 (1994).

${ }^{32}$ M. Parrinello and A. Rahman, J. Appl. Phys. 52(12), 7182 (1981).

${ }^{33}$ M. E. Tuckerman, J. Alejandre, R. Lopez-Rendon, A. L. Jochim, and G. J. Martyna, J. Phys. A 39(19), 5629 (2006).

${ }^{34}$ S. J. Plimpton, R. Pollock, and M. Stevens, paper presented at the Proceedings of the 8th SIAM Conference on Parallel Processing for Scientific Computing, Minneapolis, MN, 1997 (unpublished).

${ }^{35}$ J. J. Max and C. Chapados, J. Chem. Phys. 131(18), 184505 (2009).

${ }^{36}$ R. H. Henchman, J. Chem. Phys. 126(6), 064504 (2007); L. Wang, R. Abel, R. A. Friesner, and B. J. Berne, J. Chem. Theory Comput. 5(6), 1462 (2009).

${ }^{37}$ S. Habershon, T. E. Markland, and D. E. Manolopoulos, J. Chem. Phys. 131(2), 024501 (2009).

${ }^{38}$ M. J. McGrath, J. I. Siepmann, I. F. W. Kuo, C. J. Mundy, J. VandeVondele, J. Hutter, F. Mohamed, and M. Krack, J. Phys. Chem. A 110(2), 640 (2005).

${ }^{39}$ J. D. Cox, D. D. Wagman, and V. A. Medvedev, CODATA Key Values for Thermodynamics, Hemisphere Publishing Corp., New York, 1984, 1.

${ }^{40}$ W. Wagner and A. Pruss, J. Phys. Chem. Ref. Data 31(2), 387 (2002).

${ }^{41}$ G. S. Kell, J. Chem. Eng. Data 20(1), 97 (1975); D. Eisenberg and W. Kauzmann, The Structure and Properties of Water (Clarendon, Oxford, 1969). 